\title{
UNA NUEVA VISIÓN EN LA EDUCACIÓN SUPERIOR: TREINTA AÑOS DE LA UNIVERSIDAD ESTATAL A DISTANCIA, COSTA RICA
}

\author{
(A NEW VISION IN HIGHER EDUCATION: THIRTY YEARS OF UNIVERSIDAD ESTATAL A \\ DISTANCIA , COSTA RICA)
}

Eugenia Chaves Hidalgo

Universidad Estatal a Distancia (Costa Rica)

\section{RESUMEN}

La Universidad Estatal a Distancia de Costa Rica cumple treinta años. Sus logros en la sociedad costarricense han sido muchos y de gran trascendencia para el desarrollo del país. Tres momentos importantes han propiciado puntos de inflexión en su devenir histórico, coincidentes con las tres décadas de la institución. La primera década, desde los inicios en 1978 hasta 1989, año de creación de las escuelas; la segunda, desde 1990 hasta 1999, período de construcción de un proyecto educativo y finalmente la tercera etapa desde el 2000 hasta nuestros días, período de consolidación de este proyecto.

A la luz de una nueva visión, se propone una introspección en cada una de estas etapas, para presentar al lector cómo se construyó la respuesta educativa que la institución ofreció en cada momento.

$\mathrm{Al}$ analizar el desarrollo de la UNED, es importante mencionar el compromiso democratizador que le fue conferido en su creación y cómo la fidelidad a éste, se manifiesta en todo el recorrido histórico por las diferentes etapas de su trayectoria.

Palabras clave: monografía UNED, estudio bibliográfico de la UNED.

\begin{abstract}
UNED of Costa Rica is celebrating its $30^{\text {th }}$ anniversary. Its contributions to Costa Rican society have been many and of great importance for the growth of the country. Three important moments have provoked points of inflection in its historical development coincidental with
\end{abstract}


the three decades of the institution's life: (1) the first decade, from its beginnings in 1978 to 1989, when schools are created; (2) the second decade, from 1990 to 1999 marked by the construction of an educational project; and finally, (3) the third decade, from 2000 to the present, characterized by the consolidation of such a project.

This essay offers an introspection of every one of these stages or decades to show the reader how the institution's educational response was built in each period.

When analyzing UNED's development, it is important to emphasize the democratizing assignment given to UNED since its creation and how UNED's fidelity to this mission has been manifested throughout its history and during the different stages reviewed in this essay.

Key words: monograph, legacy of UNED.

Los artículos o investigaciones que tratan el tema de la historia de la UNED no son numerosos, de ahí que cada acción realizada para el desarrollo de este trabajo, permitió descubrir nuevos e importantes puntos de vista, todos de gran valor, ya que representaban diferentes aristas sobre hechos o sucesos relevantes en el acontecer histórico de la institución. La experiencia resultante de esta vivencia ha sido muy gratificante, volver la mirada al pasado es tratar de encontrar significado a hechos que en su momento parecían lógicos y naturales, sin embargo, nos encontramos con el coraje y la sabiduría de muchos de nuestros compañeros y compañeras que en momentos decisivos se arriesgaron para abrir una brecha y asumir con valentía la incertidumbre de sus propias decisiones ${ }^{1}$. Querer plasmar la historia de la UNED no es tarea fácil, como difícil es contar la vida institucional a lo largo de estos 30 años.

Por la gran experiencia que resulta de rememorar cada una de las etapas institucionales, agradezco a todos con quienes compartí la alegría de construir este trabajo: sus apreciaciones, sus puntos de vista, su disposición por querer contarnos sus fortalezas y sus debilidades, las cuales comprometen nuestro trabajo para seguir sus huellas. Imposible nombrar a algunos de ellos para agradecer su colaboración, porque esta institución creada de un sueño visionario de grandes hombres y mujeres, representa el esfuerzo, el cariño y el apostolado de todos los que día a día creemos en la revolución de la educación.

Para iniciar en este recorrido de la trayectoria de la UNED es necesario ubicar algunos momentos históricos que coincidentemente con los entornos, propiciaron tres etapas diferentes, cada una con características muy propias. El paso de una etapa 
a la siguiente no fue determinado por cambios bruscos que propiciaron un cambio en otra dirección. Como cualquier proceso de cambio, la adopción de políticas y la propuesta educativa institucional ha obedecido en términos generales a aquellas necesidades educativas que la UNED tuvo que atender.

Al ser esta universidad la primera en el país con una metodología basada en la educación a distancia, el camino ha sido pionero e innovador en una propuesta nueva que ha abierto opciones para estudiantes, personal académico y administrativo de la institución.

Es importante distinguir tres momentos para visualizar este camino recorrido, basándonos en uno de los aportes más relevantes de este proyecto educativo cual es la producción de los materiales didácticos. La primera etapa, como ya se mencionó, es posible ubicarla en la primera década. Desde los inicios de la institución en 1978 hasta 1989, año de creación de las escuelas. La segunda etapa abarca desde 1990 hasta 1999, período de construcción y ejecución de un proyecto educativo y finalmente la tercera desde el 2000 hasta nuestros días, que bien podría llamarse la fundamentación teórica del modelo que la institución asumió.

\section{INICIOS DE LA UNED}

El nacimiento de la UNED no responde a una sola causa, don Oscar Aguilar precursor y entusiasta del proyecto, menciona en su libro La UNED y sus orígenes que fue el entonces Ministro de Educación Fernando Volio, como resultado de la experiencia que ese momento se vivía en España con la Universidad Nacional de Educación a Distancia, el propiciador de esta idea. En sus propias palabras: "el entusiasmo de don Fernando era contagiante y sin duda veía en aquellos proyectos, como en realidad lo eran, un gran beneficio nacional" (Aguilar, 2005, p. 14).

$\mathrm{Al}$ calor de este entusiasmo se constituye la Comisión Organizadora de la UNED, la cual divide funciones en la parte jurídica, o sea el proyecto de ley y por otro la parte académica y administrativa. Finalmente, ambas corrientes del proyecto se funden en una sola dando resultado al proyecto de ley que fue presentado a la Asamblea Legislativa el 16 de mayo de 1976, firmado por el entonces Ministro Volio y el Presidente Daniel Oduber.

El camino para la aprobación no fue fácil, diversos criterios se entremezclaron como resultado de posiciones políticas encontradas, algunos más sólidos en sus planteamientos y otros más sutiles como la incorporación para que el rector 
fuera costarricense. Luego de largos meses de debate, dictámenes de las otras universidades, unos a favor y otros en contra, proyectos alternativos, el 16 de febrero de 1977 se aprobó por amplia mayoría la creación de la UNED. El 3 de marzo de 1977, al oeste del Parque Nacional, en un sencillo acto, el entonces Presidente Oduber y el Ministro Volio firmaron el ejecútese de la Ley y el 12 de marzo del mismo año, se publicó la Ley en La Gaceta, periódico oficial del gobierno de la república, para darle legalmente vida a un proyecto educativo de educación superior en Costa Rica.

En términos generales, los argumentos que justificaron su creación se pueden expresar como una solución al grave problema de financiación de la educación superior, permitiendo simultáneamente a grandes sectores de la sociedad costarricense el acceso a la educación. Se pretendía que fuera una universidad austera y con un gran potencial para el desarrollo educativo de países en desarrollo.

El 9 de abril del mismo año, se realizó la primera reunión de la Junta Universitaria con don Oscar Aguilar como secretario, don Fernando Volio como Presidente, don Federico Vargas, don Ronald García, don Chester Zelaya, don Rodrigo Gámez, don Eugenio Rodríguez, don Francisco Antonio Pacheco, don Enrique Góngora y don Walter Sagot, acordándose que el Rector sería don Francisco Antonio Pacheco, el Vicerrector Ejecutivo don Oscar Aguilar y el Vicerrector Académico don Enrique Góngora. Posteriormente se nombró como Vicerrector de Planificación a don Chester Zelaya.

El reto de la educación a distancia, plasmada ahora en un proyecto de universidad, no era una tarea fácil, no se contaba con otros modelos de referencia que sirvieran como ejemplo y más difícil aún, empezar a romper el paradigma educativo en el ambiente educativo nacional.

El diseño del proyecto, su desarrollo y posible aceptación estaba empezando. Como una de las primeras acciones se elaboró el logotipo, el cual debía expresar el mensaje de la concepción de la institución. Al respecto, Don Oscar Aguilar menciona:

"Fue obra del pintor herediano don Roberto Cambronero, acuarelista ya reconocido y con méritos en aquel momento, con el que tuvimos varias sesiones de trabajo explicándole el concepto de la UNED, a fin de que pudiera diseñar un logotipo que realmente tuviera un significado concordante con la filosofía institucional. Así, el logotipo es una U que se expande en una especie de ondas en sentido horizantal y, en lo vertical 
apuntan hacia lo superior desde diferentes niveles. Esto significa que a través de la educación a distancia y de los medios tecnológicos que se pueden utilizar, la educación superior se expande como ondas que cubren todo el territorio nacional y todos los sectores sociales, elevando al costarricense hacia universos superiores, por medio de la formación universitaria" (Aguilar, 2005, p. 35).

A partir de este momento, se inició la tarea de organizar y poner en marcha una de las mayores revoluciones educativas del país. Nuevas concepciones en el campo del proceso educativo debían abrirse paso: un concepto más amplio de las funciones del estudiante en cuanto al diseño de sus horarios de estudio y desarrollo de sus competencias como estudiante para el aprendizaje autónomo. Este nuevo concepto involucró una nueva dimensión en la forma tradicional como habíamos concebido el aprendizaje, el cual estaba basado en la lección magistral. Además y tal vez uno de los retos más difíciles, cambiar las funciones del docente para convertirlo en un mediador pedagógico.

De aquí en adelante se inicia un trabajo lleno de mística y profesionalismo basado en tres líneas de acción: el prestigio de su personal, la oportunidad de su respuesta y la calidad de sus productos.

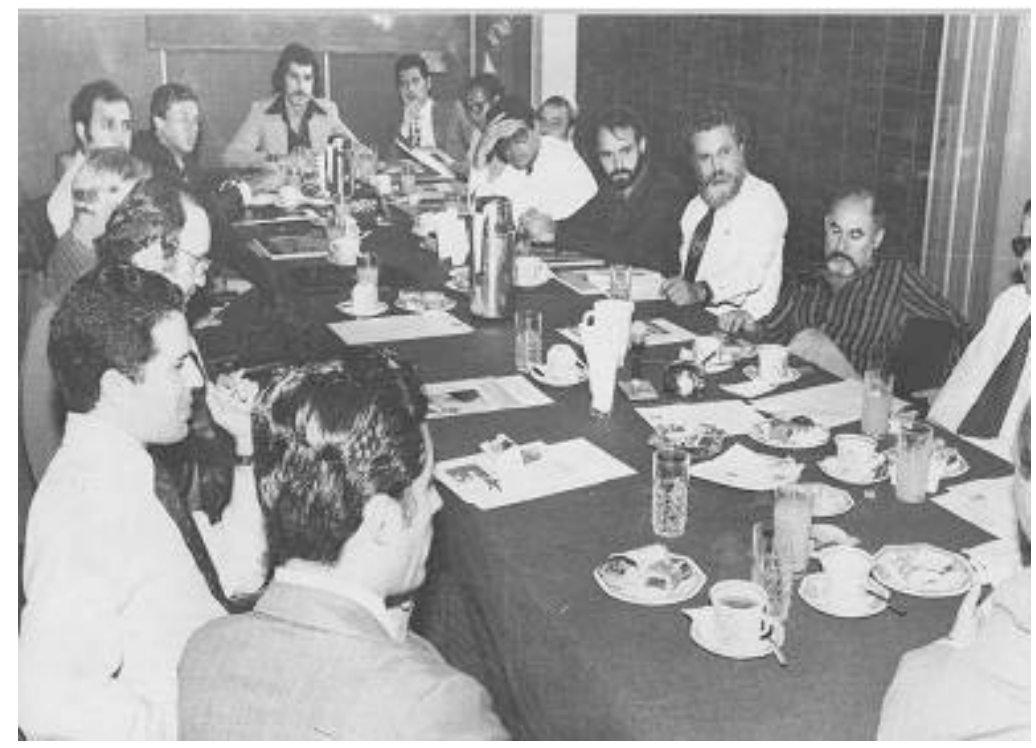

Gráfico 1- Reuniones de trabajo para la estructura de la nueva institución 
De los factores determinantes en los logros académicos de la institución, uno en especial aparece como recurrente en la mayoría de entrevistas que se realizaron para la confección de este trabajo y es justamente la primera línea de acción de la Comisión Organizadora: contratación de personal comprometido. Considerando que el proyecto era nuevo, no había otros modelos nacionales ni internacionales que sirvieran como referencia, por lo que el trabajo pionero y la creatividad fueron los derroteros de esta época.

Se estableció como política de la Comisión invitar a importantes figuras del quehacer académico nacional a participar como autores, tutores, especialistas del campo o bien en asesorías a los congresos. Entre ellas, es posible mencionar la participación de doña María Eugenia Dengo en el Taller de Planeamiento de la UNED, actividad precursora de muchas de las actividades académicas como congresos que se realizan actualmente a nivel nacional e internacional. El apoyo de doña María Eugenia a la institución se evidenció posteriormente desde su puesto como Ministra de Educación. Más adelante doña María Eugenia formó parte del Consejo Universitario de la UNED como miembro externo y es autora de unidades didácticas en el campo de la educación.

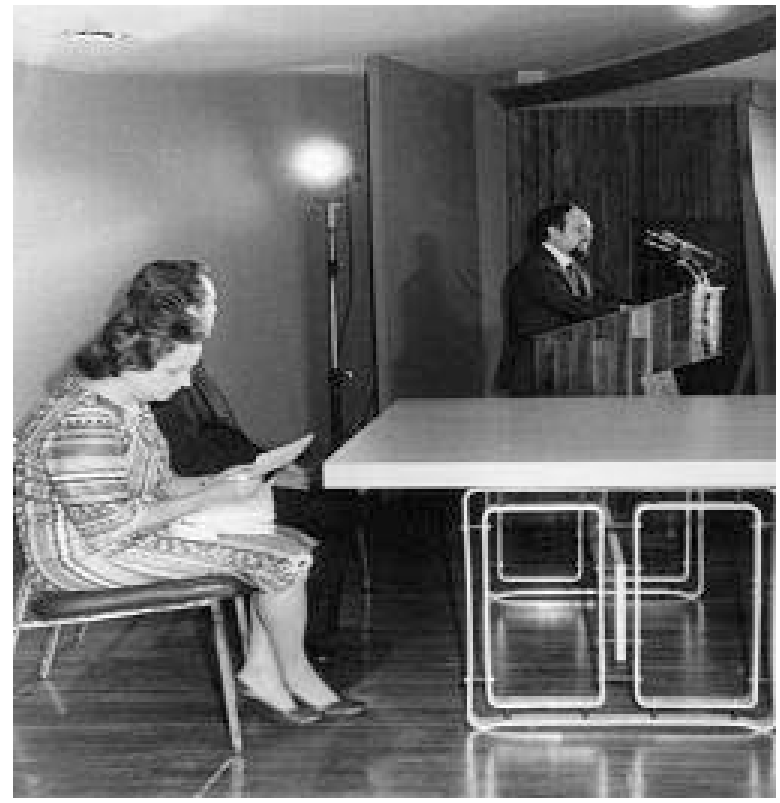

Gráfico 2 - En la gráfica doña María Eugenia Dengo, Ministra de Educación, don Rodrigo Carazo, Presidente de la República y don Francisco Antonio Pacheco, primer rector de la UNED. 


\section{ETAPA: 1978-1989}

La UNEDiniciala producción de materiales en 1979 con la Dirección de Producción Académica, época en la cuál se oficializa el primer documento con lineamientos sobre los elementos que debe tener una unidad didáctica. Con referencia a este tema, Celedonio Ramírez menciona en La Tercera Revolución que:

“A propósito de los materiales didácticos, don Enrique (Góngora) me explicó que, hemos discutido ampliamente sobre los medios de enseñanza que la UNED puede utilizar para hacer educación a distancia. Hemos rechazado la radio y la televisión, al menos temporariamente, por no contar con ellas y por ser muy caros los espacios en las radioemisoras y televisoras. Hemos considerado el posible uso de audiovisuales, pero por carecer de equipo y de expertos se ha dejado esta opción para más adelante. Como consecuencia, en la sesión n. 21 de la Junta, realizada el 28 de julio (1977) resolvimos usar como medio maestro el texto impreso, y en la sesión n. 35 del 25 de octubre decidimos que estos textos deben tener una extensión mínima de 150 páginas y una máxima de 240 páginas". (Ramírez, 2006, p. 281).

Como puede notarse, se cumple en el desarrollo de la UNED la evolución por etapas propuesta por varios autores en la cual los inicios de la educación a distancia corresponde con la producción de materiales didácticos de la primera generación o sea el medio escrito.

Con estas y otras indicaciones que aparecían en el Instructivo metodológico se elaboraron las primeras unidades didácticas que vieron la luz en mayo de 1978. Una vez elaboradas se contrató los tutores que realizarían la mediación pedagógica, llamada en aquellos tiempos, tutoría presencial y telefónica.

Esta época coincide con una fuerte corriente positivista- conductista por lo que "la producción de materiales didácticos se basa en principios como la importancia de los objetivos específicos, de los ejercicios de autoevaluación, los cuales deben permitir al estudiante autoevaluar el logro de cada uno de esos objetivos, así como el desarrollo lineal del contenido de acuerdo con los objetivos preestablecidos" (Meza, 2005, p. 1).

Posteriormente se desarrolló la producción de medios audiovisuales y materiales de video como apoyo a los materiales escritos. 
Las tutorías presenciales se apoyaban en otros materiales llamados los ATP (Ayuda para la tutoría presencial) que consistían en ejercicios de apoyo explicativos y aclaratorios de los temas de las unidades didácticas. Así se inicia un largo camino para consolidar un modelo pedagógico para la enseñanza superior a distancia.

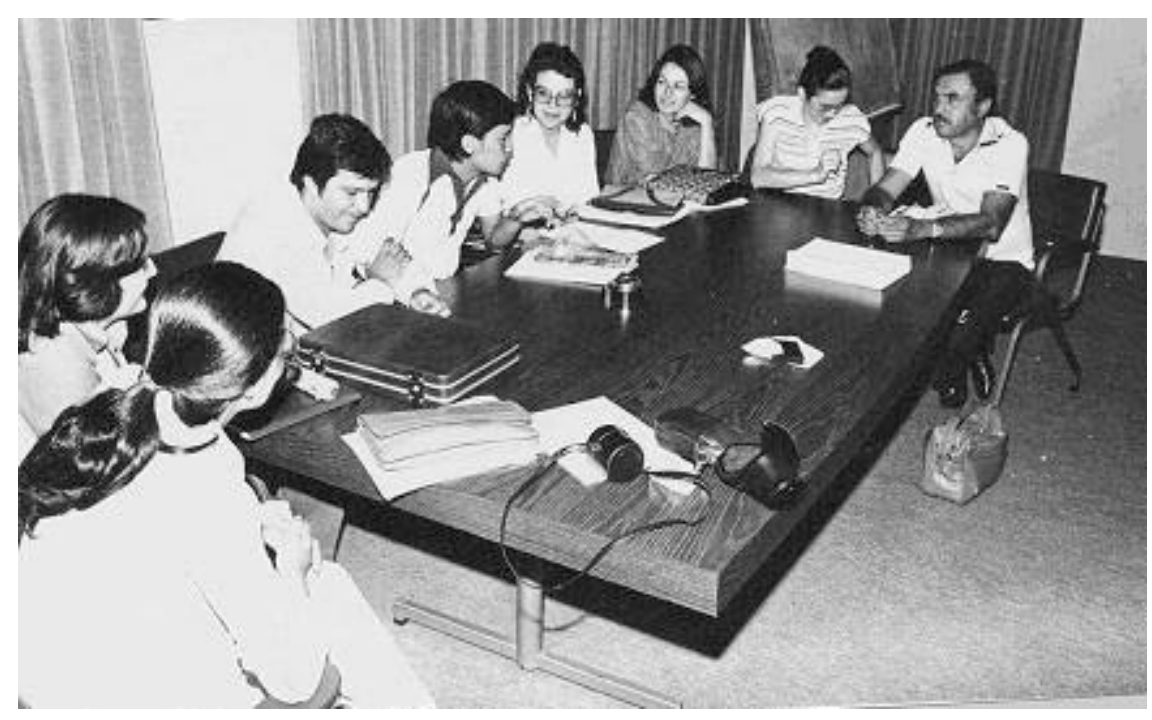

Gráfica 3 - de izquierda a derecha, Marlene Víquez, Rosario Arias, Mario Corrales, Ricardo León, María Freer, Virginia Espeleta, Eugenia Chaves y Alvaro Obando, en una reunión de coordinación de tutores de matemática.

En este camino, es importante mencionar el aporte y la asesoría del Dr. Greville Rumble, funcionario de la Open University y evaluador de la educación a distancia, quien bajo los auspicios de la Misión UNESCO realizó una investigación en los años 1979-1980, cuyos resultados fueron traducidos por Celedonio Ramírez y publicados en 1987 en la obra La UNED: una evaluación.

Esta evaluación incluyó la calidad de los materiales de enseñanza en cuanto a la ayuda en forma activa que brindaban a los estudiantes. Menciona que "la gran mayoría de textos acentúan el énfasis en los contenidos, en desmedro del esfuerzo por capacitar a los alumnos para analizar materiales, resolver problemas u ofrecer explicaciones en vez de hechos" (Rumble, 1987, p. 89). Estas condiciones expresadas hace casi 20 años mantienen su vigencia como básicas para la construcción de materiales. 
El ambiente académico propicia el Reglamento del Subsistema de Administración Académica (1984), documento en el cual se propone para los materiales didácticos: coherencia interna, secuencia lógica del discurso, estructura metodológica apta la educación a distancia, composición gramatical y ortográfica y la coherencia con la descripción curricular.

En términos generales se puede ubicar esta etapa como la puesta en marcha de un modelo educativo a distancia para la educación superior, sobre una base sólida de planificación y ejecución de sistemas.

\section{ETAPA: 1989-1999}

Esta década se encuentra inspirada en varios hechos que coincidentemente provocan y promueven un cambio en la visión futurista de la institución. El Primer Congreso Universitario, con el lema Evaluar y examinar con profundidad el papel que la educación a distancia le corresponde jugar en los próximos años en Costa Rica permite visualizar un nuevo modelo para el paquete instructivo (conjunto de materiales y otros recursos necesarios para que el alumno estudie por sí solo cada una de las asignaturas que integran el plan de estudios de una carrera). De esta forma se apunta a la conformación de equipos de trabajo para que en la interdisciplinariedad del mismo, se consideren todas las facetas del estudiante a distancia. Surge el concepto de componentes que se pueden combinar de diferentes formas para lograr materiales usables en distintos cursos. La exposición lineal da campo al desarrollo inductivo apoyado en estudio de casos y con bibliografía adicional. Este cambio se apoya en una generación de especialistas con el propósito de plantear alternativas al diseño instruccional.

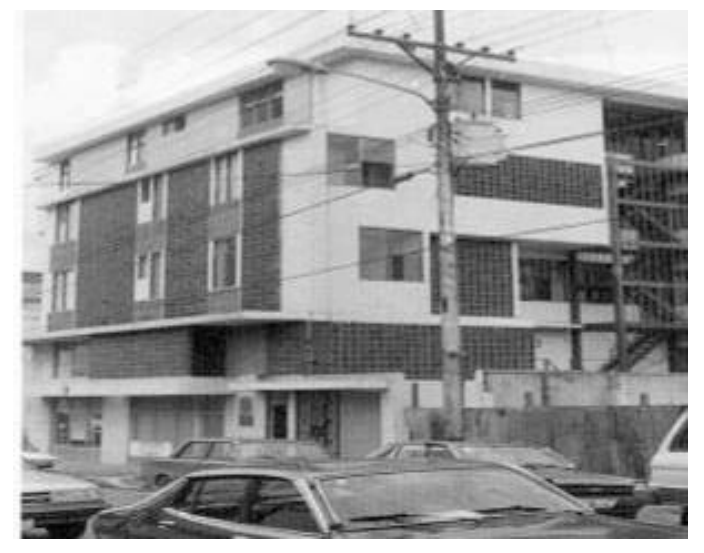

Gráfica 4 - Edificio que albergó la Dirección de Docencia. 1989. 
En 1989 se crea la Dirección de Docencia y con ello una nueva estructura acorde con las funciones docentes, se formaliza los puestos y las funciones de los tutores, encargados de cátedra y de programa, que hasta ese momento permanecían física y funcionalmente distantes. Este esquema vino a apoyar la nueva propuesta en los materiales didácticos basada en la interdisciplinariedad desde los diferentes puestos académicos relacionados con la docencia. Don Rodrigo Barrantes como Director de Docencia asume funciones con el propósito de materializar los cambios de las funciones académicas.

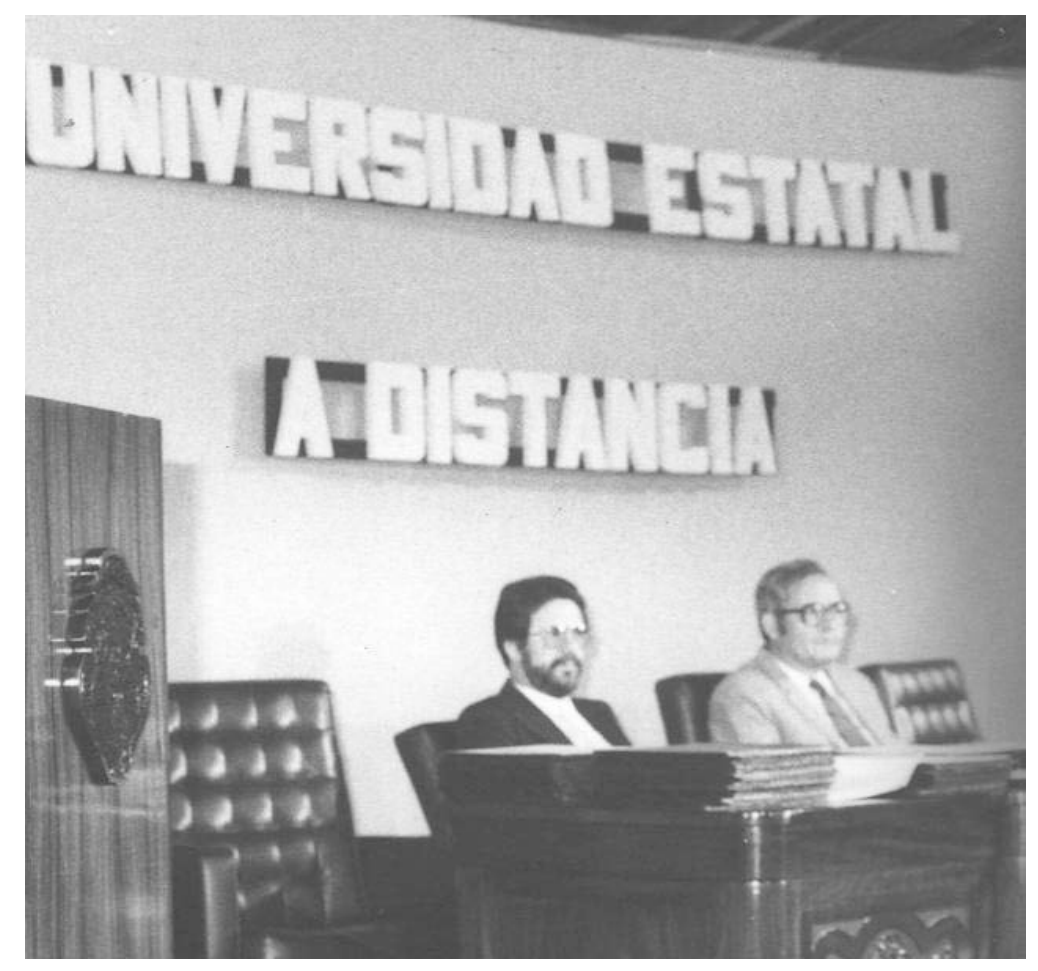

Gráfica 5 - Rodrigo Barrantes Director de Docencia y Celedonio Ramírez, Rector.

\section{ETAPA: 1999 - 2006}

Esta tercera etapa inicia con los compromisos establecidos en el nuevo Reglamento, pero más que eso, los internaliza y los replantea a la luz de las corrientes del constructivismo. En 1999, René Muiñoz publica los requerimientos 
de producción y edición de los textos didácticos. En este mismo año, se publica el Reglamento de selección de autores que norma y regula todo lo concerniente a la elaboración de unidades didácticas. En este escenario, se busca la excelencia en el discurso expositivo y la práctica docente. Surge como consecuencia de estas acciones, un repunte en la producción de materiales con autores internos de la institución, pues conocedores de una mediación pedagógica en educación a distancia, podían llevarla a la práctica en los materiales. Es la construcción de un nuevo paradigma en el cuál cambia la visión del docente, del estudiante y de la mediación pedagógica, los lectores son los constructores del conocimiento, aprenden a su ritmo y con autonomía. El material didáctico es el medio que ayuda en la construcción del saber promoviendo la creatividad, la interdisciplinariedad y la interactividad.

En los Lineamientos de Política Institucional, aprobados en el 2001, se visualiza la institución como gestora del conocimiento con una función de creación, reelaboración y democratización del conocimiento. Para ello, dedica la sección II Democratización y excelencia académica: Partiendo del derecho que poseen todos los seres humanos de participar en pie de igualdad en los procesos culturales, la UNED se perfila frente a los otras instituciones de educación superior, por su deber ineludible de asegurar que este derecho sea una realidad; es decir, que la desigualdad en las condiciones reales de vida de las personas, generadas por un sistema social y económico esencialmente injusto y excluyente, no genere una correspondiente desigualdad en el acceso a la educación.

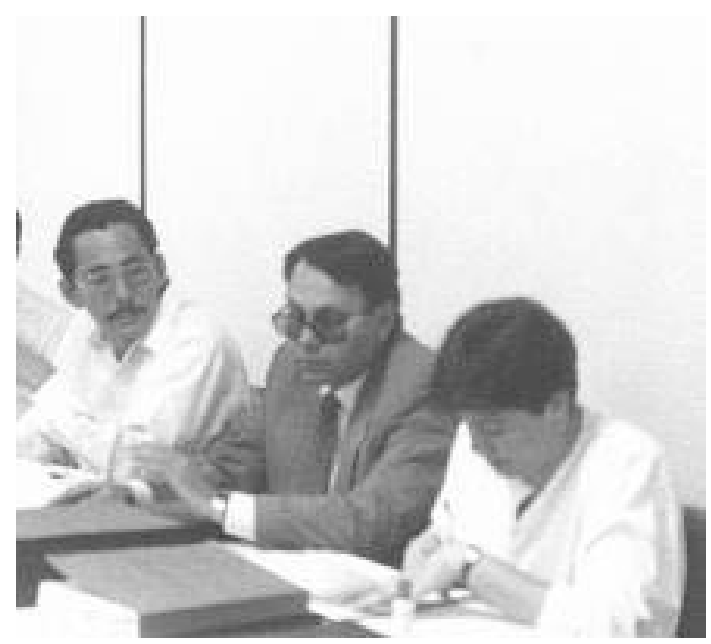

Gráfico 6 - Personal académico de la Escuela Ciencias de la Educación en una tesis: en el orden usual Walter Solano, Beltrán Lara y Cecilia Balmaceda. 
En el II Congreso Universitario: por un cambio con participación real, se visualiza un cambio, como realmente lo menciona el tema del congreso, sobre el compromiso democratizador de la institución. La moción 003 señala que

"El concepto de democratización de la educación superior será entendido en la UNED, como el acceso, la permanencia y el éxito del estudiante dentro de su sistema de educación a distancia y la garantía de la calidad de la oferta académica. Además la democratización debe comprender al ámbito de la docencia y expresarse, en la medida de lo posible, por la vía de la extensión en sus actividades de difusión y capacitación no formal; y por la vía de la investigación aplicada a la realidad nacional y a las necesidades de los sectores sociales menos favorecidos en nuestro país".

En los inicios de la institución, en la Ley de Creación, artículo 2, inciso (c) se menciona "incorporar a la educación superior, con métodos idóneos y flexibles, a quienes no hubieran podido incorporarse al sistema formal universitario".

El II Congreso aprueba que el éxito del estudiante ya no debe limitarse a la incorporación a la educación superior, incluye su permanencia y su éxito ligado a la calidad de la oferta académica. Una visión más amplia e integral del accionar del estudiante.

El efecto democratizador de esta etapa se manifiesta en todas las acciones del quehacer docente. Dos importantes documentos se discuten en el 2004, el Modelo Pedagógico y el Reglamento de la Gestión Académica. El Modelo pedagógico se aprueba finalmente en julio del 2004, sienta las bases de la construcción teórica y práctica de la educación a distancia, conservando de los orígenes los planteamientos de base. Coloca al estudiante como centro del modelo por lo que el proceso educativo debe proporcionar todos lo medios disponibles para que pueda planificar el progreso de su avance. En la última sección incluye las recomendaciones metodológicas para la producción de unidades didácticas modulares, con las siguientes características para la comunicación didáctica:

- Tener una estructura clara y explícita.

- Permitir que el estudiante comprenda yhaga propias las metas de aprendizaje, facilitándole establecer relaciones con sus experiencias previas, intereses y expectativas.

- Desarrollar los contenidos con rigurosidad académica y con una estructura didáctica de la cual el estudiante pueda apropiarse. 
- Favorecer la recuperación de los conocimientos previos pertinentes del alumno.

- Partir de núcleos temáticos propios de la realidad profesional, y, de ser posible, relacionados con el contexto del estudiante y con el mundo del ejercicio práctico de la profesión, de manera que el estudiante le encuentre sentido al conocimiento y lo proyecte funcionalmente.

- Utilizar ejemplos, metáforas y el pensamiento analógico, tan propio del aprendiz adulto.

- Mantener un nivel de flexibilidad que permita, al estudiante, el dominio sobre los procesos para lograr las metas propuestas, respetando los diversos estilos de aprendizaje.

- Incorporar actividades que exijan del alumno procesos de pensamiento crítico, reflexivo y creativo.

- Incorporar actividades de autorregulación, las cuales exigen que el estudiante se apropie de los criterios con los que se juzgará su desempeño, y habilidades de auto-observación y planificación de las propias acciones de estudio.

- Utilizar una estructura flexible que posibilite la conformación de un cuerpo de conocimientos teóricos y prácticos que mantengan su vigencia en el tiempo.

Este modelo pedagógico como bien se expresa en los considerandos de aprobación, es una respuesta a los acuerdos del II Congreso Universitario y a los Lineamientos de Política Institucional y concretiza los principios y fundamentos del quehacer académico institucional.

Una vez establecidos estos principios y fundamentos se requiere una estructura funcional y dinámica que organice el accionar académico. Hasta el momento aún se encontraba vigente el Reglamento del subsistema de administración académica, por lo que era necesario actualizar los procesos y procedimientos de la gestión académica. Este nuevo documento se llamó Reglamento de la Gestión Académica y es aprobado por el Consejo Universitario en marzo del 2006.

Este reglamento presenta como gestión académica los siguientes procesos:

- Investigaciones y evaluaciones que orienten la oferta académica, su viabilidad y desarrollo

- Planeamiento y programación curricular

- Producción de los materiales didácticos

- Ejecución del proceso educativo 
- Evaluación de carreras, programas, cursos, materiales didácticos y de la gestión académica.

Los dos reglamentos ya mencionados y los acuerdos del Consejo Universitario han orientado la solidez del modelo educativo de la institución en esta etapa. En cuanto a la investigación el Consejo Universitario aprueba en el 2002, "el desarrollo de la investigación para la generación, renovación, ampliación y aplicación del conocimiento, además para reforzar, innovar y asegurar la calidad de los programas académicos. La investigación científica debe promoverse desde todas las unidades académicas en particular desde las Escuelas y los Centros Universitarios”. Con este acuerdo se organiza, articula y promueve la investigación desde todos los ámbitos universitarios.

En esta etapa se han desarrollado otros programas que involucran nuevos y diferentes apoyos a los estudiantes haciendo uso de los avances tecnológicos como la videoconferencia. Este programa se inicia en el 2000 y ha representado una opción para crear espacios de comunicación e interacción entre estudiantes y profesores a nivel nacional e internacional. Se ha constituido como la primera red en el país para promover el aprendizaje. Actualmente cuenta con gran cantidad de salas de videoconferencia propias de la UNED y algunas otras por convenio con otras instituciones.

La creación del Sistema de Estudios de Posgrado abrió nuevas posibilidades para la continuación de estudios superiores en diferentes áreas. Los estudios universitarios en posgrado inician en la UNED en 1998, con dos maestrías y han continuado su implementación hasta llegar a la oferta actual.

El Sistema de Estudios de Posgrado (SEP) es:

"La unidad académico-administrativa encargada de gestionar los programas de posgrado a distancia en coordinación con las diferentes escuelas académicas de la institución. Implementa los programas que las escuelas identifican como necesarios para el impulso del desarrollo cultural, educativo y socioeconómico de Costa Rica y los demás países del área centroamericana. El desarrollo de los programas se orienta hacia la especialización de profesionales en diferentes disciplinas del saber, con un enfoque transdisciplinario y orientación transcultural. Se apoya, además, en la investigación para contribuir a generar conocimientos y a reformular conceptos. Los graduados del SEP son profesionales que pueden tomar 


\section{E. CHAVES \\ Una Nueva Visión en la Educación Superior: Treinta Años de la Universidad Estatal a Distancia, Costa Rica}

una distancia crítica frente a los procesos de cambio y asumir una actitud protagónica, dentro de un marco ético y de sensibilidad" de la misión del SEP.

Para realizar esta misión se tiene la siguiente visión: el SEP llevará una oferta académica de calidad a las comunidades profesionales e internacionales con énfasis estratégico en los países centroamericanos. Los programas se consolidarán conformando equipos profesionales de alto nivel y con el uso racional de las tecnologías que faciliten y mejoren los procesos de enseñanza-aprendizaje. Se concibe la estructura del SEP como un sistema en el cual la orientación académica está dirigida por las escuelas a las que pertenecen los programas y al Sistema de Estudios de Posgrado en cuanto a su organización, impulso y administración. Los posgrados se desarrollarán en las unidades académicas en las cuales fueron aprobados.

Actualmente se tiene en oferta cuatro doctorados: Ciencias de la Administración, Ciencias Naturales para el Desarrollo, Derecho y Educación.

A nivel de maestrías se tiene 20 programas académicos en los más diversos campos.

La Editorial de la Universidad se crea el 13 de octubre de 1979 por un acuerdo de la Junta Universitaria. Hoy, 30 años después se mantiene como una de las editoriales más exitosas de Centro América. La producción e impresión de materiales, desde su creación, abrió un espacio para que autores nacionales, literatos costarricenses, intelectuales, artistas, publicaran textos didácticos y obras culturales, acción que ha irrumpido en el mercado de la producción con gran calidad.

En el plano externo, la visión de su trabajo se concibe como "la consolidación de ese papel de ser la mayor editorial universitaria de Centroamérica, que en los próximos años no solamente se va a consolidar como la más productiva editorial de la región, sino también como la que asume un papel de liderazgo en la producción editorial de universidades centroamericanas. Esto va ligado con las políticas de internacionalización de la Universidad, para que se complemente con la apertura de librerías de la UNED en Centroamérica" (Arias, 2005, p. 70).

\section{FINANCIAMIENTO}

En materia del financiamiento de la institución, es importante mencionar que la UNED, como parte de las universidades estatales, es parte del Consejo Nacional de Rectores lo que garantiza ingresos como parte de la subvención que el Estado 
otorga a este Consejo. Como parte de este consejo, el artículo 85 de la Constitución Política manifiesta que: "el Estado dotará de patrimonio propio a la Universidad de Costa Rica, el Instituto Tecnológico de Costa Rica, la Universidad Nacional y a la Universidad Estatal a Distancia, les creará rentas propias, independientemente de las originadas en estas instituciones. Además mantendrá con las rentas actuales y con otras que son necesarias un fondo especial para el lineamiento de la Educación Superior Estatal”.

En el 2005 por iniciativa del actual rector, se somete a consideración de la Asamblea Legislativa la incorporación de la UNED a la Ley 8459 para abrir otras fuentes de financiamiento. La aprobación unánime de esta Ley, se traduce como el reconocimiento que la sociedad hace a una institución de gran trayectoria, formadora de profesionales y que con un nuevo modelo pedagógico, ha sabido abrirse paso con excelencia y calidad en sus quehaceres académicos.

\section{DESARROLLO TECNOLÓGICO}

En cuanto al desarrollo tecnológico, se han incorporado las diferentes tecnologías de la información y la comunicación al modelo educativo para facilitar la comunicación didáctica con los estudiantes. Asimismo, le ha permitido a los docentes incursionar en formas o alternativas para crear opciones pedagógicas acordes con los diferentes contextos educativos de la educación a distancia.

Como respuesta a esta realidad, se han abierto espacios para llegar a diversos sectores con una respuesta educativa acorde con los tiempos actuales. En esta disyuntiva, no se ha olvidado que algunos sectores sólo tienen acceso al material impreso, pero no por eso, debe limitarse su aprendizaje.

En los diferentes Centros Universitarios se han abierto posibilidades para que los estudiantes tengan acceso a equipo de cómputo y con ello, conocer y valorar las posibilidades que Internet ofrece. La puesta en marcha de una nueva plataforma tecnológica abre un abanico de posibilidades para la apertura de cursos en línea.

\section{RETOS DE LA UNED}

A treinta años de trabajo, la UNED se ha consolidado como la primera y única universidad a distancia del país. Pionera en su campo está obligada a mantener esa condición de liderazgo, para ello muchos son los retos que al mismo tiempo 
se constituyen en estímulos y desafíos académicos. Los resultados alcanzados se materializan en la formación y capacitación de profesionales, en la construcción de materiales didácticos, y en la conducción de un proceso de reforma educativa a nivel superior mediante el compromiso por democratizar el acceso, permanencia y éxito de todos a la educación. Así, "La educación a distancia se posiciona entonces, como un modelo educativo innovador, transformador y prospectivo en cuanto a lo que debía ser el desarrollo educativo para el siglo XXI” (Arias, 2006).

Muchos son los retos que se presentan a este modelo educativo y a la UNED como institución con más experiencia en esta modalidad educativa. En el Tercer Congreso Universitario 2006, "por el fortalecimiento de una academia con calidad" se plantearon una serie de acciones inmediatas para construir una plataforma académica que oriente los pasos de la institución en los próximos años. Derivados de ésta y otras acciones institucionales podemos mencionar algunos de los temas expuestos para el próximo quinquenio:

- Educación y tecnología, son factores claves en la construcción de una sociedad más justa, equitativa, en la cual el acceso al conocimiento no sea una limitante.

- La investigación debe estar referida a las actividades para la generación, renovación, ampliación y aplicación del conocimiento. Debe existir articulación con la sociedad para que los procesos de investigación ayuden y contribuyan en el mejoramiento de la sociedad.

- La extensión como actividad inherente a la docencia, es un compromiso con todos los sectores sociales.

- Los cursos en línea no deben construirse en un escenario que promueva las brechas digitales. Como impulsores de una democratización del conocimiento, parte de este reto es el acceso, la permanencia y el éxito del estudiante.

- La capacitación constante de los profesionales académicos para adaptar las posibilidades de la tecnología a ambientes de aprendizaje innovadores. "La UNED como universidad tiene el compromiso de producir un cuerpo de profesionales altamente capacitados y de enriquecer el acervo científico y tecnológico pertinente" (Hidalgo, 2003, p. 37).

- El estudiante debe desarrollar competencias para el aprendizaje mediado por ambientes virtuales para lograr una inserción real y efectiva en la sociedad. 


\section{NOTAS}

1 Para la realización de este trabajo se entrevistó a diversos funcionarios, jubilados algunos, con el propósito de obtener puntos de vista y criterios de hechos históricos quellegaron a convertirse en hitos para el desarrollo de esta institución. Las entrevistas realizadas, así como materiales y fotos, se encuentran en poder de la autora como material de referencia y consulta para futuras investigaciones.

\section{REFERENCIAS BIBLIOGRÁFICAS}

Aguilar, O. (2005). La UNED y sus orígenes. San José, Costa Rica: EUNED.

Arias, R (2006). Discurso de inauguración. En: XIII Congreso Internacional sobre tecnología y educación a distancia. San José, Costa Rica: EUNED.

EUNED (2005). Nuestros libros tienen la palabra. Memoria. San José, Costa Rica: EUNED.

Hidalgo, G. (2003). El rol de la UNED en la transición hacia la comunidad global de la sociedad costarricense. RIED, 6(1).

Meza, J. (2004). Etapas del desarrollo y planteamientos actuales en la producción de los materiales impresos de la Universidad Estatal a Distancia (UNED). San José, Costa Rica: MIMEO.
UNED (2001). Lineamientos de política institucional 2001-2006. Rumble, G. (1987). La UNED una evaluación. San José, Costa Rica: EUNED.

UNED (2006). Estatuto Orgánico. San José, Costa Rica: UNED.

UNED (2004). Modelo Pedagógico. San José, Costa Rica: UNED.

UNED (2005). Reglamento de la gestión Académica de la UNED. San José, Costa Rica: UNED.

UNED (2002). Acuerdos tomados en sesión 1560-2002. Propuesta sobre el desarrollo de la investigación en la UNED. San José, Costa Rica: UNED.

\section{PERFIL ACADÉMICO Y PROFESIONAL DE LA AUTORA}

Eugenia Chaves Hidalgo, Doctora en Educación de la Universidad Estatal a Distancia, Licenciada en enseñanza de la matemática de la Universidad de Costa Rica. Se desempeña como profesora investigadora de la Escuela Ciencias de la Educación, UNED. Ha sido profesora universitaria y directora de la Escuela Ciencias de la Educación por cinco años. Actualmente desarrolla proyectos de investigación para la maestría en educación a distancia y en el área de competencias universitarias del profesional académico universitario.

E-mail: echaves@uned.ac.cr 


\section{E. CHAVES}

Una Nueva Visión en la Educación Superior: Treinta Años de la Universidad Estatal a Distancia, Costa Rica

DIRECCIÓN DE LA AUTORA

Eugenia Chaves Hidalgo

Universidad Estatal a Distancia

Apartado 474-2050 San Pedro de Montes de Oca

Costa Rica

Fecha recepción del artículo: 26/01/07

Fecha aceptación del artículo: 05/03/07 